\title{
Correction to: Solving the Initial Value Problem for the 3-Wave Interaction Equations in Multidimensions
}

\author{
M. C. van der Weele ${ }^{1} \cdot$ A. S. Fokas ${ }^{1,2}$
}

Published online: 11 April 2021

(c) Springer-Verlag GmbH Germany, part of Springer Nature 2021

\section{Correction to: Computational Methods and Function Theory https://doi.org/10.1007/s40315-020-00357-2}

In the last paragraph of Sect. 3 the last sentence "Hence, these time-dependent $\mu_{a n}$ 's are given by Eq. (2.9) with the $f_{a b}$ 's replaced by the corresponding $h_{a b}$ 's of Eq. (3.3)." has to be replaced by

Hence, these time-dependent $\mu_{a n}$ 's are given by Eq. (2.39) with the $f_{a b}$ 's replaced by the corresponding $h_{a b}$ 's of Eq. (3.3).

In the first paragraph of Sect. 4 the part "In the linear limit we have, as can be inferred from Eq. (2.39)." of the sentence before Eq. (4.1) has to be replaced by

In the linear limit we have, as can be inferred from Eq. (2.9).

The original article can be found online at https://doi.org/10.1007/s40315-020-00357-2.

M. C. van der Weele

mcv26@cam.ac.uk

1 Department of Applied Mathematics and Theoretical Physics, University of Cambridge, Cambridge CB3 0WA, UK

2 School of Engineering, University of Southern California, Los Angeles, California 90089, USA 